\title{
Structural Brain Network Measures in Elderly Patients with Cerebral Small Vessel Disease and Depressive Symptoms
}

\section{Yumeng Gu}

Second Hospital of Tianjin Medical University

\section{Ping Zhao}

Second Hospital of Tianjin Medical University

\section{Wenjun Feng}

Second Hospital of Tianjin Medical University

\section{Xiaoshuang Xia}

Second Hospital of Tianjin Medical University

\section{Xiaolin Tian}

Second Hospital of Tianjin Medical University

\section{Yu Yan}

Second Hospital of Tianjin Medical University

\section{Xiaowen Wang}

Second Hospital of Tianjin Medical University

\section{Decheng Gao}

Second Hospital of Tianjin Medical University

\section{Yanfen Du}

Second Hospital of Tianjin Medical University

Xin Li (D lixinsci@126.com)

Second Hospital of Tianjin Medical University

\section{Research Article}

Keywords: Cerebral small vessel disease, depression, diffusion tensor imaging, structural network, tractbased spatial statistics

Posted Date: February 16th, 2022

DOI: https://doi.org/10.21203/rs.3.rs-1278822/v1

License: (c) (1) This work is licensed under a Creative Commons Attribution 4.0 International License. Read Full License 


\section{Abstract}

Objectives: To investigate whether there is a difference in the incidence of depression between patients with small cerebral vascular disease (CSVD) and normal controls (NC), and the relationship between depression and white matter fiber damage and structural brain network in elderly patients with CSVD.

Method: A total of 115 elderly subjects were consecutively recruited from the neurology clinic, including 36 patients with CSVD with depressive state, 34 patients with CSVD without depressive state, and 45 NCs. A detailed neuropsychological assessment and multimodal MRI were performed. Based on TBSS analysis and structural network analysis, differences between groups were compared, and logistic regression was used to determine the predictive value of TBSS indicators and structural brain network measures for depression in CSVD patients.

Results: Group differences were found in global TBSS analysis and brain network measures. After adjusting for age, gender, education level, and vascular risk factors (VRF), there were significant correlations between TBSS indicators and structural network indicators and depression, including: FA ( $r=$ $-0.291, p<0.05), E_{\text {Local }}(r=-0.278, p<0.01)$ and in network strength $(r=-0.403, p<0.001)$. At the same time, $E_{\text {Local }}$ was an independent risk factor for depression in patients with CSVD.

Conclusions: Changes in FA, MD values and structural network indicators in DTI parameters can predict the depressive state of CSVD to a certain extent, providing a more direct structural basis for the hypothesis of abnormal neural circuits in the pathogenesis of vascular depression. In addition, we believe that abnormal white matter alterations in subcortical neural circuits affect the microstructural function of brain connections, which may be a mechanism for the concomitant depressive symptoms in CSVD patients.

\section{Introduction}

With the gradual increase in the number of elderly people in China, cardiovascular and cerebrovascular diseases are frequent, and the incidence of CSVD is also on the rise. The characteristics of high morbidity, disability, mortality and recurrence rate have brought different degrees of damage to social development and personal health, and are accompanied by different degrees of physical and psychological disorders [1]. CSVD is an important cause of vascular-related depression [2, 3]. Given that the underlying pathogenesis remains controversial [4], multimodal neuroimaging is usually used to detect the lesions leading to CSVD. There are many recognized CSVD structural markers on MRI [5], including white matter hyperintensity $(\mathrm{WMH})$, lacunae, cerebral microbleeds (CMBs), and enlarged perivascular spaces (EPVS), etc. These features of CSVD imaging are interrelated, and their correlation with depression has been proved [6, 7].

With the continuous development of multimodal neuroimaging technology, the understanding of brain information processing tends to construct interconnected holistic brain network analysis methods. Currently, many studies have shown that brain network connections in CSVD patients are disrupted [8-10], 
the DTI technique provides a method to study white matter fiber disconnection damage and damage to structural connections of brain regions [11]. White matter microstructural damage caused by CSVD destroys the integrity of white matter fibers and also damages the nerve fiber network, resulting in symptoms such as psychomotor retardation, hypomotility, low mood, and cognitive decline [12]. The damage of brain white matter microstructure caused by CSVD is related to psychomotor retardation, hypomotility, low mood, and cognitive decline [12]. However, whether these new findings will be identified in CSVD with depressive symptoms has not been determined.

Therefore, we aimed to explore the relationship between white matter microstructure and depression in patients with CSVD by using different DTI measurements. By comparing the relationship between TBSS indicators and structural brain network measures in CSVD patients, to provide some evidence for the potential mechanism of early depression in CSVD. In addition, we aimed to search for more downstream neuroimaging markers in patients with CSVD-induced depression.

\section{Methods}

\subsection{Participants}

We recruited 115 right-handed elderly subjects from the Neurology Department of the Second Hospital of Tianjin Medical University. The inclusion criteria of CSVD patients were as follows: (1) 50 to 80 years old; (2) at least 6 years of education; (3) Meet Staals scoring criteria [13]; (4) Informed consent signed by participants. All CSVD patients underwent overall psychological function through Montreal Cognitive Assessment (MoCA). Patients were assessed for depression using the 17 item Hamilton Depression Rating Scale (HAMD-17). Patients with a HAMD-17 score of 7-17 were classified as CSVD+D, while those with a score below 7 were classified as CSVD-D [14-16].

Exclusion criteria: (1) severe physical disability; (2) WMH due to non-vascular dysfunction; (3) Cognitive impairment; (4) Clinical dementia score (CDR) < 0.5; (5) MoCA score < 26; (6) No stenosis of intracranial and extracranial vascular; (7) Cardiogenic cerebral embolism; (9) Alcohol or drug abuse disorder or serious mental disorder; (10) Inability to cooperate with evaluation or MRI.

Forty-five healthy NCs were included from Tianjin, China. The inclusion criteria of NC are as follows: (1) No clinical stroke history; (2) at least 6 years of education; (3) No history of major diseases of liver, heart, lung and other important organs; (4) Fazekas grade $\leq 1$ and MRI scan showed no other significant abnormalities; (5) HAMD-17 score < 8; (6) Cognitive assessment test were normal; (7) No vascular risk factors such as hypercholesterolemia, diabetes, hypertension, and smoking evidence.

During the course of the study, 3 CSVD patients were excluded for personal reasons, 2 controls and 2 CSVD patients for visible head movement. Therefore, 36 CSVD+D patients, 34 CSVD-D patients and 45 NCs were included. All subjects gave written informed consent, and this study was approved by the institutional review board of the second hospital of Tianjin Medical University. 
MRI scans were performed on a 3.0 T scanner (GE HealthCare, USA). T1 images parameters: TR $=8.2 \mathrm{ms,}$ $\mathrm{TE}=3.2 \mathrm{~ms}, \mathrm{TI}=450 \mathrm{~ms}$, slice thickness $=1.0 \mathrm{~mm}$, gap $=0$, flip angle $=12^{\circ}, \mathrm{FOV}=256 \times 256 \mathrm{~mm}^{2}$, slice number $=166 ;$ T2 FLAIR images parameters: $\mathrm{TE}=150 \mathrm{~ms}, \mathrm{TR}=9,075 \mathrm{~ms}, \mathrm{TI}=2,250 \mathrm{~ms}$, FOV $=256 \mathrm{~mm} 2$, number of slices $=160$; DTI sequences parameters: $\mathrm{TR}=8,000 \mathrm{~ms}, \mathrm{TE}=88.4 \mathrm{~ms}$, matrix $=128 \times 128$, FOV $=256 \times 256 \mathrm{~mm}, \mathrm{NEX}=1$, slice thickness $=2 \mathrm{~mm}$, gap $=0, \mathrm{~b}$ value is $1000 \mathrm{~s} / \mathrm{mm}^{2}$ and b0 $=0$.

\subsection{Grading and Evaluation of CSVD Lesions on MRI}

The total burden of CSVD imaging was reflected using a well validated CSVD burden scoring scale, which is an ordinal scoring scale of 0-4 points. One point when there is one or more lacunar lesions (LI) on MRl; one point when there are deep microbleeds (CMBs); one point when 3 scores on the Fazekas scale for periventricular white matter hyperintensities (WMHs) and (or) 2-3 scores on the Fazekas scale of centrum semiovale WMHs; one point when the grade of enlarged perivascular space (EPVs) in basal ganglia is grade 2-3 [13]. The number of CMBs was scale $d$ by Microbleed anatomical grading scale (MARS) [17]. Maclulich's method was used to record and score EPVS [18]. The above scores were rated by two experienced neurologists blinded to clinical data.

\subsection{MRI Data Preprocessing Analysis}

\subsubsection{Structural Data}

Structural images were preprocessed using the CAT12 toolbox available in the Statistical Parametric Mapping 12 package (SPM12). The following steps were used through MATLAB 2019a: normalized T1 images to template space and segmented images into gray matter, white matter, and cerebrospinal fluid; visual inspection; the total intracranial volume was evaluated; check for data homogeneity; the Gaussian kernel of full width at half maximum $6 \mathrm{~mm}(\mathrm{FWHM}=6 \mathrm{~mm})$ was used. The "brain volume/ intracranial volume (ICV) x 100\%" was used to display WMHs, gray and white matter volume [19].

\subsubsection{DTI Measures}

DTI images were pre and post processed using FSL software and analyzed based on TBSS [20]. Skeleton threshold set to less than 0.2 . The skeletonized FA or MD maps were entered into a voxel group level analysis. Statistical significance was assessed using the FSL permutation test. As the statistical modeling of voxel wise statistical analysis the general linear model (GLM) was performed. Voxel-based pairwise group comparisons of CSVD-D versus CSVD+D, NC versus CSVD+D, and NC versus CSVD-D were performed using GLM. The covariates including sex, age, education, and VRF. The significance level of the two DTI parameters (FA, MD) is $\mathrm{P} \otimes 0.05$ to illustrate the difference between the groups, displayed using the JHU white matter tractography atlas.

\subsubsection{Brain Network Construction}


Using the Pipeline of the Analyze Brain Diffusion Image Toolkit (PANDA) to preprocess the raw DTI data [21]. Using the automated anatomical labeling (AAL) 90 atlas [22].The Fiber Assignment Continuous Tracking (FACT) algorithm was used to generate whole brain white matter fiber reconstructions. Streamlines terminate if the fiber is switched on at an angle $>45^{\circ}$ or encounters a voxel with FA $<0.2$ [23]. The structured brain network was ultimately structured by 90 for each subject $90 \times 90$ FN weighted matrix construction. Using the graph theoretic network analysis toolbox (GRETNA) [24], global topology metrics were calculated. Different rarefaction thresholds ranging from 0.1 to 0.3 at 0.01 intervals. The topological property parameters analyzed in this study include: global efficiency $\left(\mathrm{E}_{G \mid o a l}\right)$, local efficiency $\left(E_{\text {Local }}\right)$, network strength and small world properties. The network strength was defined as the overall accumulation of $\mathrm{FN}$ on all nodes [25].

\subsection{Demographic and Clinical Measurements}

Analyses were performed with SPSS 19.0 (Chicago, IL), baseline data were analyzed by one-way analysis of variance (ANOVA). Normally distributed measurement data are expressed as $\mathrm{x} \pm \mathrm{s}$. The number of use cases (percentage) for categorical data is expressed, comparisons between groups were performed using the $\chi^{2}$ test. Post hoc t-test was used for comparisons between groups, and Bonferroni correction was used for multiple comparisons. Partial correlation analysis was used to assess the relationship between Hamilton Depression Score and DTI measures in patients with CSVD after adjusting for age, sex, education, and VRF. Two-tailed p-value $<0.05$ was considered statistically significant.

\section{Result}

\subsection{Demographic Data and Clinical Measurements}

Among the subjects $(n=115), 70$ subjects were diagnosed with CSVD, of which 36 CSVD patients were diagnosed with the presence of depression status (CSVD+D), the remaining 34 CSVD patients were without depression status (CSVD-D), and 45 subjects were normal controls (NC). The baseline data are listed in Table 1. There were no significant differences in age, gender, education and MoCA scores among the three groups $(P>0.05)$. There were no significant differences in VRF between the CSVD-D and $C S V D+D$ groups $(P>0.05)$. The HAMD scores of CSVD+D group were the highest among the three groups, representing the most severe depression. HAMD scores were significantly lower in NC group compared to CSVD-D and CSVD+D groups.

\subsection{Neuroimaging Findings in Patients with CSVD}

There were no significant differences in terms of CSVD burden scores between the CSVD-D and CSVD+D groups, imaging findings, and brain volume (as a percentage of total intracranial volume), and the results are shown in Table 2. The results of group comparisons of the three DTI measurement indexes are shown in Fig. 1. FA, MD, $E_{G l o b a l}, E_{L o c a l}$, and network strength showed significant differences among the three groups. The values of FA, $E_{\text {Global, }} E_{\text {Local }}$ and network intensity were significantly lower in CSVD+D than in 
CSVD-D and NC groups. Meanwhile, MD was significantly higher in CSVD+D than in CSVD-D and NC groups (Bonferroni corrected, $\mathrm{P}<0.05$ ).

\subsection{FA and MD of Whole-brain Fiber Tracts}

For evaluating voxel WM integrity differences between each group pair using the TBSS method to analyze DTI parameters (Fig. 2 and Fig. 3). Compared with the CSVD-D group, the CSVD+D group showed significantly decreased FA values and significantly elevated MD values in a wide range of WM tracts. Compared with the NC group, the CSVD+D group showed significantly decreased FA values and significantly increased MD values in extensive WM tracts. Meanwhile, compared with the NC group, the CSVD-D group shows significantly decreased FA values in a wide range of WM tracts. The extensive WM tracts described above, including: Anterior thalamic radiation, bilateral Corticospinal tract, bilateral cingulate gyrus, bilateral hippocampus $\mathbb{F}$ Forceps major, Forceps minor, bilateral Inferior fronto-occipital fasciculus, bilateral Inferior longitudinal fasciculus, bilateral Superior longitudinal fasciculus, bilateral Uncinate fasciculus, and bilateral Superior longitudinal fasciculus (temporal part) (FWE corrected, $\mathrm{P}<$ 0.05). However, there was no significant difference in MD values between CSVD-D group and NC group.

\subsection{Differences in Structural Network Indicators}

The NC group, CSVD-D group, and CSVD+D group showed good small world properties at all thresholds ( $\mathrm{Y}$ $>1, \lambda \approx 1, \sigma>1)$. We analyzed the differences in the structural network of the three groups shown in Figures 4,5,6. Compared with CSVD-D group, CSVD+D group had obviously damaged nodes, mainly located in left frontal_mid and left Insula (Bonferroni correction, $P<0.05$ ). Compared with the NC group, the nodes with significantly impaired efficiency in the CSVD+D group were mainly located in bilateral Hippocampus, bilateral frontal_mid_orb, bilateral frontal_mid, bilateral insula, bilateral frontal_sup, right olfactory, right fusiform, right frontal_sup_orb, right amygdala and left lingual (Bonferroni correction, P < 0.05). Compared with the NC group, the CSVD-D group had significantly impaired nodes, mainly located in left caudate, left pallidum, left frontal_mid_orb, left frontal_sup_orb, right amygdala, right frontal_inf_orb, right olfactory (Bonferroni correction, $\mathrm{P}<0.05$ ).

\subsection{Correlation Analysis}

Between DTI measures and depression scale scores adjusted for age, gender, education and VRF the partial correlation analysis was performed. We chosed FA to represent fiber tract measurements because FA and MD were based on the same principle and FA was the most commonly used indicator for fiber tract measurements. A significant correlation was found between FA and HAMD scores $(r=-0.291, P<$ $0.05), E_{\text {Local }}$ and HAMD scores $(r=-0.278, P<0.01)$ and was also found between network strength and HAMD scores $(r=-0.403, P<0.001)$.

However, no significant correlation was observed between $E_{\text {Global }}$ and HAMD scores $(r=-0.142, P=0.136)$.

\subsection{Logistic Regression Analysis}


To explore whether DTI measurements could predict depressive status in patients with CSVD the multiple logistic regression analysis was applied. FA, $\mathrm{E}_{\text {Local, }}$ and network strength were all input into the regression model, at the same time, age, gender, education, and VRF were adjusted for potential confounders. Taked the NC group as a control, only $\mathrm{E}_{\text {Local }}$ was significantly correlated with CSVD and depressive status, while FA and network strength showed no significant relationship, as shown in Table 3.

\section{Discussion}

We investigated DTI sequences based on TBSS analysis methods to identify damage to white matter fibers during the disease process. The results indicate that CSVD patients who develop depression state, white matter fiber tracts were damaged. For FA and MD, compared with the NC group and the CSVD-D group, the CSVD+D group showed different degrees of damage to fiber tracts in almost all parts of the brain. Notably, there was no statistical difference between NC group and CSVD-D group in terms of MD, which means that in patients with CSVD and depression, while demyelination of nerve fibers occurs, there is also significant damage to the microstructural organization. FA measures the restriction of the movement of water molecules in all directions, and can describe the histomorphological features of the region and decreased FA is associated with disturbed white matter fiber homogeneity [26]. Some studies reported that MD may be more sensitive in detecting mild damage, while FA captures more severe damage $[27,28]$. However, some studies have also reported opposite results, in which FA may be more sensitive to detect disease changes [29]. Long associated fibers such as superior longitudinal fasciculus and uncinate fasciculus showed a significant increase in MD in the preclinical stage. These longassociated fibers are generally considered to form myelin relatively late and are vulnerable to injury and decline in later life [30]. Disruption of white matter integrity may be related to changes in the structure and density of nerve fibers, as well as decreased axonal numbers, abnormal myelin sheaths, and gliosis [31], this change may be the neuroanatomical basis of CSVD with depression, which reflects the hypothesis of

"vascular depression" to a certain extent [32]. Then, the damaged cingulate gyrus found in this study is an important part of the emotional circuit and participates in the processes of emotion and self-evaluation, which are closely related to depressive symptoms. Some scholars have found that there are abnormalities in cerebral blood flow and metabolism in the posterior cingulate gyrus of patients with depression, which suggest that depression may have low function of the posterior cingulate gyrus [33].

The significant finding of this study is that the characteristics of structural network are closely related to depression in patients with CSVD. Because the structural brain network represents the integrity of white matter connections, it arguably reflects the underlying mechanisms of depression in patients with CSVD more than any other measure. The structural network connectivity of the CSVD-D group was significantly higher than that of the CSVD+D group. The present study found that, with regard to $\mathrm{E}_{\text {Nodal }}$, the NC group showed significantly higher than CSVD+D group in several areas, including: bilateral hippocampus, bilateral frontal_mid_orb, bilateral frontal_mid, bilateral insula, bilateral frontal_sup, right olfactory, right fusiform, right frontal_sup_orb, right amygdala and left lingual, most of these areas are part of the limbic system. Limbic cortex includes allogenic cortex (such as the ancient cortex of the hippocampus and 
dentate gyrus, the septum and the amygdala, etc.) and the intermediate cortex (including the posterior part of the orbitofrontal cortex, insula, temporal pole, cingulate gyrus, etc.), participate in mediating instinctual and emotional behavior [34]. At the same time, neuroanatomy has confirmed that the prefrontal lobe is the key part of emotion regulation, which is related to cognition, emotion and conscious experience. Regarding the hippocampus found in this study, it is mainly responsible for memory and emotional control. Studies have shown that patients with recurrent and persistent depression have significantly smaller hippocampus in the brain. However, no changes in this part of the depression were found in the primary stage, which means that the disease itself caused this change in the brain. However, the study also pointed out that this change is reversible, because the shrinkage is mainly due to the disconnection of nerve cells, rather than the death of nerve cells as in dementia. The combination of psychological and drug therapy can promote hippocampal regrowth [35]. We also studied non-depressed CSVD patients as well as healthy controls. The results showed that DTI measurements deteriorated gradually from NC to CSVD, followed by concomitant depression.

Possible mechanisms of the pathogenesis of depressive symptoms in CSVD: 1. "Vascular depression hypothesis" holds that the increased incidence of depression in CSVD may be due to the destruction of subcortical pathway connecting brain regions caused by cerebral white matter lesions caused by cerebrovascular diseases, namely prefrontal subcortical circuit, this suggests that structural damage inherently disrupts network efficiency, leading to a depressive state [32,36]; 2. CSVD is destructive when it occurs, it disrupts the cortico-striatal-pallidum-thalamocortical circuit, affects noradrenergic and serotonergic neural pathways, resulting in a decrease in the levels of these two neurotransmitters, the subsequent corresponding metabolic activities are hindered, inability to achieve normal physiological activities, and then resulting in depression [37]; 3 . Cerebral ischemia leads to the occurrence of oxidative stress, free radicals can attack the brain nervous tissue, cause astrocyte apoptosis, interfere with the tissue maintenance of the brain by microglia and the repair of neural damage, which is also an important reason for the occurrence of depression related network damage [38, 39].

\section{Limitations}

There are still deficiencies in this study: First, the sample size of our study was relatively small, although we felt that the study was sufficiently effective for the analysis presented. Second, a cross-sectional cohort study and cannot draw a causal relationship between depressive states and brain structural changes, thus requiring repeated measures longitudinal data. Third, deterministic fiber tracking has some limitations in tracking complex white matter structures, especially cross fibers, which will be improved by more advanced white matter imaging technology.

\section{Conclusion}

In this study, DTI technology was applied to elderly patients with CSVD and depression. At the same time, the degree of depression was evaluated by HAMD score, FA, MD values and structural network indexes in patients' DTI parameters were effectively measured. Through data comparison and analysis, the results 
suggest that FA, MD and / or structural network indexes change in emotion related brain areas (frontotemporal lobe, thalamus, cingulate gyrus and hippocampus), which revealed that CSVD patients with depression have damage to the integrity of brain white matter. The change of white matter microstructure and structural network index can reflect the degree of depression to a certain extent, provides a more direct structural basis for the hypothesis of abnormal neural circuits in the pathogenesis of vascular depression.

\section{Declarations}

\section{Ethics approval and consent to participate}

This study was approved by the ethics committee of The Second Hospital of Tianjin Medical University. All participants have signed an informed consent form. All methods involving human participants were performed in accordance with the ethical standards of the institutional research committee and the 1964 Helsinki declaration and its later amendments or comparable ethical standards.

\section{Consent for publication}

Not applicable.

\section{Availability of data and materials}

The datasets generated and/or analysed during the current study are not publicly available due to the complexity and uniqueness of the raw MRI data, as well as the limitations of laboratory policies but are available from the corresponding author on reasonable request.

\section{Competing interests}

The authors declare that they have no competing interests.

\section{Funding}

Funding of the study was provided by the National Key Research and development Plan "New Strategy on Pathogenesis and Clinical Diagnosis and Treatment of Chronic Cerebral Small Vessels" (No. 2016YFC1300600).

\section{Authors' contributions}

YG and $X L$ designed the study, WF, PZ, $X T$ and $Y D$ helped screen and included subjects, $Y G, Y Y, X W$ and DG collected and built the database, $Y G$ and $X W$ processed the analysis data, $Y G$ and $P Z$ wrote the manuscript, $X L$ and $X X$ reviewed and revised manuscript and all authors read and approved the final version of the submitted manuscript.

\section{Acknowledgements}


The authors thank all participants for their support.

\section{References}

1. Pantoni L: Cerebral small vessel disease: from pathogenesis and clinical characteristics to therapeutic challenges. The Lancet Neurology 2010, 9(7):689-701.

2. Allan CL, Sexton CE, Kalu UG, McDermott LM, Kivimäki M, Singh-Manoux A, Mackay CE, Ebmeier KP: Does the Framingham Stroke Risk Profile predict white-matter changes in late-life depression? International psychogeriatrics 2012, 24(4):524-531.

3. Bunce D, Batterham PJ, Christensen H, Mackinnon AJ: Causal associations between depression symptoms and cognition in a community-based cohort of older adults. The American journal of geriatric psychiatry : official journal of the American Association for Geriatric Psychiatry 2014, 22(12):1583-1591.

4. Rensma SP, van Sloten TT, Launer LJ, Stehouwer CDA: Cerebral small vessel disease and risk of incident stroke, dementia and depression, and all-cause mortality: A systematic review and meta-analysis. Neuroscience and biobehavioral reviews 2018, 90:164-173.

5. Wardlaw JM, Smith EE, Biessels GJ, Cordonnier C, Fazekas F, Frayne R, Lindley RI, O'Brien JT, Barkhof F, Benavente $\mathrm{OR}$ et al: Neuroimaging standards for research into small vessel disease and its contribution to ageing and neurodegeneration. The Lancet Neurology 2013, 12(8):822-838.

6. Brookes RL, Herbert V, Lawrence AJ, Morris RG, Markus HS: Depression in small-vessel disease relates to white matter ultrastructural damage, not disability. Neurology 2014, 83(16):1417-1423.

7. Pasi M, Poggesi A, Salvadori E, Diciotti S, Ciolli L, Del Bene A, Marini S, Nannucci S, Pescini F, Valenti R et al: White matter microstructural damage and depressive symptoms in patients with mild cognitive impairment and cerebral small vessel disease: the VMCl-Tuscany Study. International journal of geriatric psychiatry 2016, 31(6):611-618.

8. Ter Telgte A, van Leijsen EMC, Wiegertjes K, Klijn CJM, Tuladhar AM, de Leeuw FE: Cerebral small vessel disease: from a focal to a global perspective. Nature reviews Neurology 2018, 14(7):387-398.

9. Yi L, Wang J, Jia L, Zhao Z, Lu J, Li K, Jia J, He Y, Jiang C, Han Y: Structural and functional changes in subcortical vascular mild cognitive impairment: a combined voxel-based morphometry and resting-state fMRI study. PloS one 2012, 7(9):e44758.

10. Li M, Meng Y, Wang M, Yang S, Wu H, Zhao B, Wang G: Cerebral gray matter volume reduction in subcortical vascular mild cognitive impairment patients and subcortical vascular dementia patients, and its relation with cognitive deficits. Brain and behavior 2017, 7(8):e00745.

11. Lope-Piedrafita S: Diffusion Tensor Imaging (DTI). Methods in molecular biology (Clifton, NJ) 2018, 1718:103-116. 
12. Kloppenborg RP, Nederkoorn PJ, Grool AM, Vincken KL, Mali WP, Vermeulen M, van der Graaf Y, Geerlings MI: Cerebral small-vessel disease and progression of brain atrophy: the SMART-MR study. Neurology 2012, 79(20):2029-2036.

13. Staals J, Makin SD, Doubal FN, Dennis MS, Wardlaw JM: Stroke subtype, vascular risk factors, and total MRI brain small-vessel disease burden. Neurology 2014, 83(14):1228-1234.

14. Riedel M, Möller HJ, Obermeier M, Schennach-Wolff R, Bauer M, Adli M, Kronmüller K, Nickel T, Brieger $\mathrm{P}, \mathrm{Laux} \mathrm{G}$ et al: Response and remission criteria in major depression-a validation of current practice. Journal of psychiatric research 2010, 44(15):1063-1068.

15. Zimmerman M, Martinez JH, Young D, Chelminski I, Dalrymple K: Severity classification on the Hamilton Depression Rating Scale. Journal of affective disorders 2013, 150(2):384-388.

16. Pavlovic AM, Pekmezovic T, Zidverc Trajkovic J, Svabic Medjedovic T, Veselinovic N, Radojicic A, Mijajlovic M, Tomic G, Jovanovic Z, Norton M et al: Baseline characteristic of patients presenting with lacunar stroke and cerebral small vessel disease may predict future development of depression. International journal of geriatric psychiatry 2016, 31(1):58-65.

17. Gregoire SM, Chaudhary UJ, Brown MM, Yousry TA, Kallis C, Jäger HR, Werring DJ: The Microbleed Anatomical Rating Scale (MARS): reliability of a tool to map brain microbleeds. Neurology 2009, 73(21):1759-1766.

18. Maclullich AM, Wardlaw JM, Ferguson KJ, Starr JM, Seckl JR, Deary IJ: Enlarged perivascular spaces are associated with cognitive function in healthy elderly men. Journal of neurology, neurosurgery, and psychiatry 2004, 75(11):1519-1523.

19. Huang L, Chen X, Sun W, Chen H, Ye Q, Yang D, Li M, Luo C, Ma J, Shao P et al: Early Segmental White Matter Fascicle Microstructural Damage Predicts the Corresponding Cognitive Domain Impairment in Cerebral Small Vessel Disease Patients by Automated Fiber Quantification. Frontiers in aging neuroscience 2020, 12:598242.

20. Smith SM, Jenkinson M, Johansen-Berg H, Rueckert D, Nichols TE, Mackay CE, Watkins KE, Ciccarelli O, Cader MZ, Matthews PM et al: Tract-based spatial statistics: voxelwise analysis of multi-subject diffusion data. Neurolmage 2006, 31(4):1487-1505.

21. Cui Z, Zhong S, Xu P, He Y, Gong G: PANDA: a pipeline toolbox for analyzing brain diffusion images. Frontiers in human neuroscience 2013, 7:42.

22. Tzourio-Mazoyer N, Landeau B, Papathanassiou D, Crivello F, Etard O, Delcroix N, Mazoyer B, Joliot M: Automated anatomical labeling of activations in SPM using a macroscopic anatomical parcellation of the MNI MRI single-subject brain. Neurolmage 2002, 15(1):273-289. 
23. Taoka T, Morikawa M, Akashi T, Miyasaka T, Nakagawa H, Kiuchi K, Kishimoto T, Kichikawa K: Fractional anisotropy-threshold dependence in tract-based diffusion tensor analysis: evaluation of the uncinate fasciculus in Alzheimer disease. AJNR American journal of neuroradiology 2009, 30(9):17001703.

24. Wang J, Wang X, Xia M, Liao X, Evans A, He Y: GRETNA: a graph theoretical network analysis toolbox for imaging connectomics. Frontiers in human neuroscience 2015, 9:386.

25. Du J, Wang Y, Zhi N, Geng J, Cao W, Yu L, Mi J, Zhou Y, Xu Q, Wen W et al: Structural brain network measures are superior to vascular burden scores in predicting early cognitive impairment in post stroke patients with small vessel disease. Neurolmage Clinical 2019, 22:101712.

26. Grieve SM, Williams LM, Paul RH, Clark CR, Gordon E: Cognitive aging, executive function, and fractional anisotropy: a diffusion tensor MR imaging study. AJNR American journal of neuroradiology 2007, 28(2):226-235.

27. Cubon VA, Putukian M, Boyer C, Dettwiler A: A diffusion tensor imaging study on the white matter skeleton in individuals with sports-related concussion. Journal of neurotrauma 2011, 28(2):189-201.

28. O'Dwyer L, Lamberton F, Bokde AL, Ewers M, Faluyi YO, Tanner C, Mazoyer B, O'Neill D, Bartley M, Collins DR et al: Multiple indices of diffusion identifies white matter damage in mild cognitive impairment and Alzheimer's disease. PloS one 2011, 6(6):e21745.

29. Bosch B, Arenaza-Urquijo EM, Rami L, Sala-Llonch R, Junqué C, Solé-Padullés C, Peña-Gómez C, Bargalló N, Molinuevo JL, Bartrés-Faz D: Multiple DTI index analysis in normal aging, amnestic $\mathrm{MCl}$ and AD. Relationship with neuropsychological performance. Neurobiology of aging 2012, 33(1):61-74.

30. Bender AR, Völkle MC, Raz N: Differential aging of cerebral white matter in middle-aged and older adults: A seven-year follow-up. Neurolmage 2016, 125:74-83.

31. Takeda M, Tanaka T, Kudo T: Elderly depression and diffusion tensor imaging. Psychogeriatrics : the official journal of the Japanese Psychogeriatric Society 2011, 11(1):1-5.

32. Alexopoulos GS, Meyers BS, Young RC, Campbell S, Silbersweig D, Charlson M: 'Vascular depression' hypothesis. Archives of general psychiatry 1997, 54(10):915-922.

33. Martin SD, Martin E, Rai SS, Richardson MA, Royall R: Brain blood flow changes in depressed patients treated with interpersonal psychotherapy or venlafaxine hydrochloride: preliminary findings. Archives of general psychiatry 2001, 58(7):641-648.

34. Murray I, Bhanot G, Bhargava A: Neuron-Glia-Immune Triad and Cortico-Limbic System in Pathology of Pain. Cells 2021, 10(6). 
35. Hermens DF, Hatton SN, Lee RSC, Naismith SL, Duffy SL, Paul Amminger G, Kaur M, Scott EM, Lagopoulos J, Hickie IB: In vivo imaging of oxidative stress and fronto-limbic white matter integrity in young adults with mood disorders. European archives of psychiatry and clinical neuroscience 2018, 268(2):145-156.

36. Tuladhar AM, van Norden AG, de Laat KF, Zwiers MP, van Dijk EJ, Norris DG, de Leeuw FE: White matter integrity in small vessel disease is related to cognition. Neurolmage Clinical 2015, 7:518-524.

37. Loubinoux I, Kronenberg G, Endres M, Schumann-Bard P, Freret T, Filipkowski RK, Kaczmarek L, PopaWagner A: Post-stroke depression: mechanisms, translation and therapy. Journal of cellular and molecular medicine 2012, 16(9):1961-1969.

38. Wang Q, Jie W, Liu JH, Yang JM, Gao TM: An astroglial basis of major depressive disorder? An overview. Glia 2017, 65(8):1227-1250.

39. Jha MK, Lee WH, Suk K: Functional polarization of neuroglia: Implications in neuroinflammation and neurological disorders. Biochemical pharmacology 2016, 103:1-16.

\section{Tables}

TABLE 1 | Demographic and clinical characteristics 


\begin{tabular}{|lllll|}
\hline & NC & CSVD-D & CSVD+D & $p$-value \\
& $n=45$ & $n=34$ & $n=36$ & \\
\hline Demographic factors & & & & \\
\hline Male (\%) & $20(44.4)$ & $19(55.9)$ & $19(52.8)$ & 0.569 \\
\hline Age, mean (SD) & $63.33(6.03)$ & $63.06(5.22)$ & $63.42(6.53)$ & 0.966 \\
\hline Education, mean (SD) & $10.60(2.42)$ & $10.56(2.14)$ & $10.25(2.64)$ & 0.788 \\
\hline Vascular risk factors & & & & \\
\hline Hypercholesterolemia (\%) & - & $8(23.5)$ & $9(25.0)$ & 0.886 \\
\hline Diabetes Mellitus (\%) & - & $7(20.6)$ & $12(33.3)$ & 0.231 \\
\hline Hypertension (\%) & - & $17(50.0)$ & $20(55.6)$ & 0.642 \\
\hline Smoking (\%) & - & $17(50.0)$ & $17(47.2)$ & 0.816 \\
\hline Drinking (\%) & - & $15(44.1)$ & $16(44.4)$ & 0.978 \\
\hline Neuropsychological tests & & & & \\
\hline MoCA, mean (SD) & $27.80(1.47)$ & $27.59(1.58)$ & $27.42(1.38)$ & 0.513 \\
\hline HAMD, mean (SD) & $1.91(0.99)$ & $2.29(1.61)$ & $11.53(2.66)$ & $<0.001^{\mathrm{a}, \mathrm{b}, \mathrm{c}}$ \\
\hline
\end{tabular}

Data represent number (percentage) and mean (standard deviation) for normally distributed data; SD, standard deviation; $p$-value $<0.05$ was considered to be statistically significant; Abbreviations: CSVD, cerebral small vessel disease; CSVD-D, CSVD without depression symptoms; CSVD+D with depression symptoms; NC, normal control; MOCA, Montreal Cognitive Assessment; HAMD, Hamilton Depression Rating Scale; a-c: post hoc analysis revealed the source of ANOVA (a: NC vs. CSVD-D, b: NC vs. CSVD+D, and C: CSVD-D vs. CSVD+D).

TABLE 2 | Neuroimaging findings of CSVD patients 


\begin{tabular}{|llll|}
\hline & $\begin{array}{l}\text { CSVD-D } \\
n=34\end{array}$ & $\begin{array}{l}\text { CSVD+D } \\
n=36\end{array}$ & $p$-value \\
\hline Cerebrovascular lesions & & & \\
\hline CSVD score, median (IQR) & $1(1)$ & $1.5(1)$ & 0.415 \\
\hline Lacunes occurrence (\%) & $12(35.3)$ & $18(50)$ & 0.214 \\
\hline CMBs occurrence (\%) & $8(23.5)$ & $9(25)$ & 0.886 \\
\hline Fazekas score, median (IQR) & $2(2)$ & $2(1)$ & 0.403 \\
\hline EPVS occurrence (\%) & $3(8.8)$ & $2(5.6)$ & 0.596 \\
\hline Neuroimaging measures & & & \\
\hline Total GMV, mean (SD) & $32.81(2.33)$ & $32.74(2.11)$ & 0.896 \\
\hline Total WMV, mean (SD) & $26.94(3.21)$ & $27.39(2.56)$ & 0.435 \\
\hline Total WMHV, mean (SD) & $3.14(1.95)$ & $4.01(2.27)$ & 0.091 \\
\hline
\end{tabular}

Data represent mean \pm standard deviation or median (interquartile range). * means P-value $<05$ was considered to be statistically significant. Abbreviations: CSVD, cerebral small vessel disease; CSVD-D, CSVD without depression symptoms; CSVD+D with depression symptoms; NC, normal control; CMBs, cerebral microbleeds; WMH score, white matter hyperintensity score; EPVS, enlarged perivascular spaces; TIV, total intracranial volume; Total GMV, gray matter volume as \% of TIV; Total WMV, white matter hyperintensity as \% of TIV; Total WMHV, white matter hyperintensity volume as \% of TIV; SD, standard deviation; IQR: interquartile range.

TABLE 3 | Multiple Logistic Regression Analysis

\begin{tabular}{|llll|}
\hline Variate & NC & CSVD-D & CSVD+D \\
OR $(95 \% \mathrm{Cl})$ & $n=45$ & $n=34$ & $n=36$ \\
\hline FA & 1 & $2.093(0.495,8.851)$ & $2.053(0.471,8.949)$ \\
\hline E $_{\text {Local }}$ & 1 & $0.206(0.057,0.749)^{\star}$ & $0.209(0.056,0.778)^{\star}$ \\
\hline Network strength & 1 & $1.327(0.477,3.689)$ & $0.697(0.224,2.163)$ \\
\hline
\end{tabular}

Data represent odds ratio (95\% confidence interval) for normally distributed data; SD, standard deviation; *, p-value $<0.05$ was considered to be statistically significant. Model: Adjusted gender, age, education and MoCA. Abbreviations: CSVD, cerebral small vessel disease; CSVD-D, CSVD without depression symptoms; CSVD+D with depression symptoms; NC, normal control; MoCA, Montreal Cognitive Assessment; $\mathrm{OR}$, odds ratio; $\mathrm{Cl}$, confidence interval. 

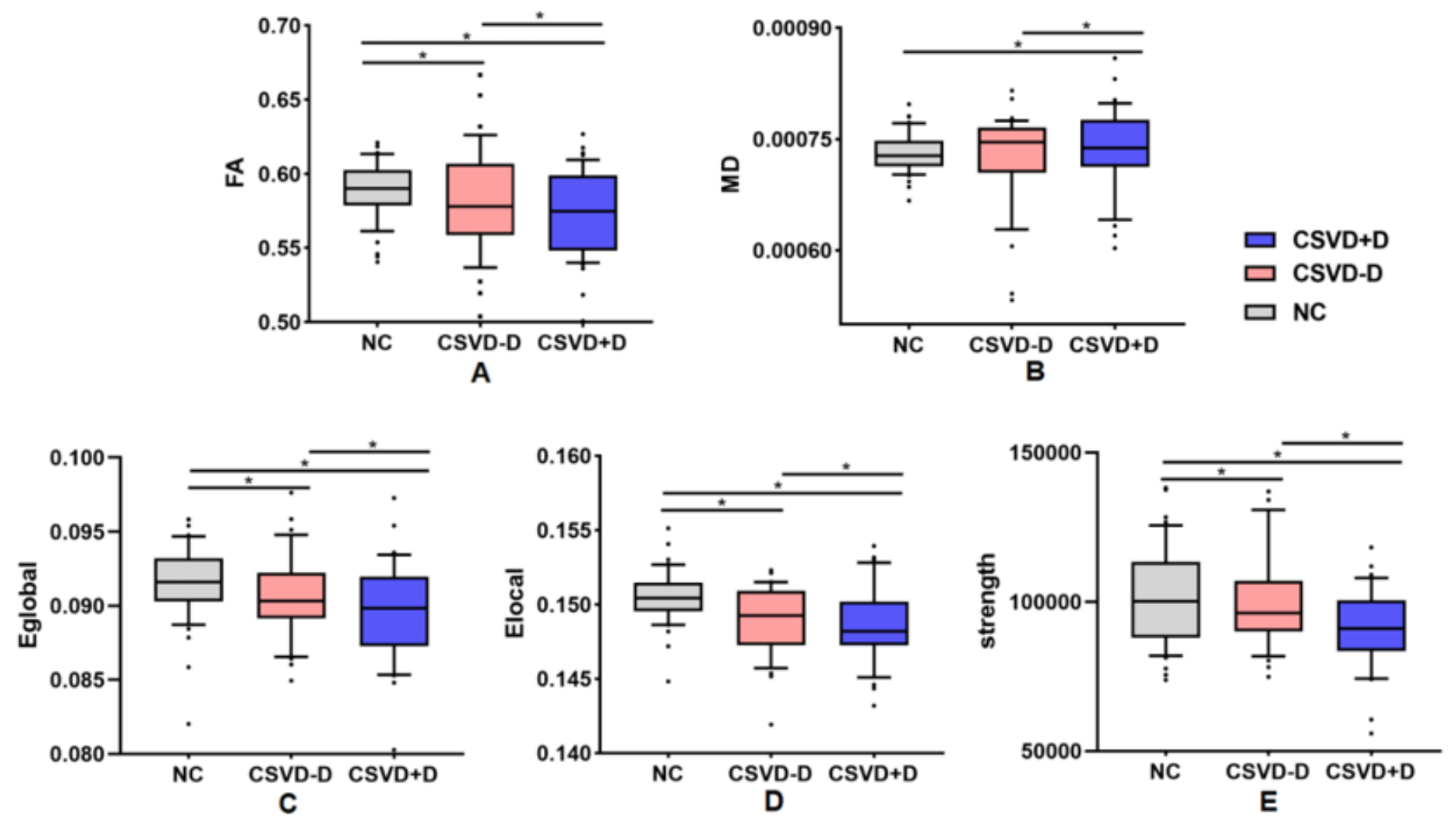

Figure 1

Group difference of DTI measures. (A-E) One-way ANOVA test was applied to conduct the group comparison of DTI measures, $p$-value $<0.05$ was considered to be statistically significant. * represents $p$ value $<0.05$ after Bonferroni correction.

Abbreviations:CSVD-D, CSVD without depression symptoms; CSVD+D with depression symptoms; NC, normal control; FA, mean fractional anisotropy of the skeleton; MD, mean of mean diffusivity of the skeleton; Eglobal , global efficiency; Elocal , local efficiency. 


\section{Figure 2}

Voxel-wise Tract-Based Spatial Statistics differences in FA metrics between groups. Green represents the mean WM skeleton of all subjects. Top row: Red-yellow voxels (thickened for better visibility) represent the WM regions with decreased FA in the CSVD+D patients compared with CSVD-D subjects (FWE corrected, $P<0.05)$. Middlerow: Red-yellow voxels represent the WM regions with reduced FA in the CSVD+D group compared with HC group (FWE corrected, $\mathrm{P}<0.05$ ). Bottom row: Red-yellow voxels represent the WM regions with reduced FA in the CSVD-D group compared with HC group (FWE corrected, $\mathrm{P}<0.05)$. 


\section{Figure 3}

Voxel-wise Tract-Based Spatial Statistics differences in MD metrics between groups. Green represents the mean WM skeleton of all subjects. Top row: bule voxels (thickened for better visibility) represent the WM regions with increased MD in the CSVD+D patients compared with CSVD-D subjects (FWE corrected, $P<0.05)$. Middlerow: bule voxels represent the WM regions with increased MD in the CSVD+D group compared with $\mathrm{HC}$ group (FWE corrected, $\mathrm{P}<0.05$ ). Bottom row: There are no significant WM microstructural differences between the CSVD-D patients and HC subjects. 
$\mathbf{L}$
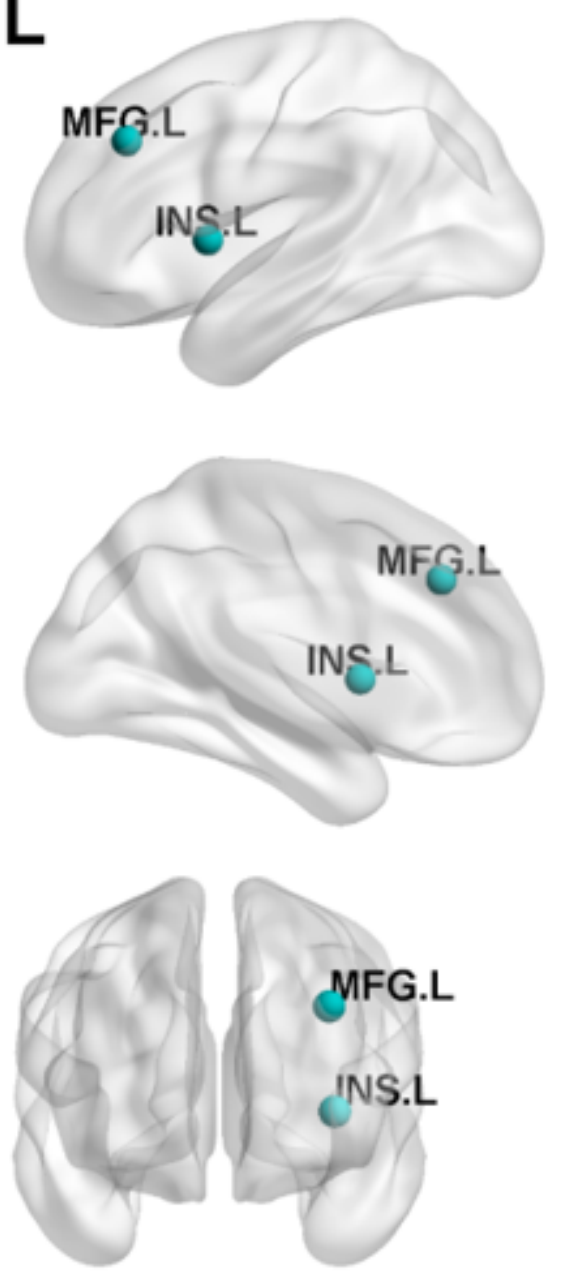
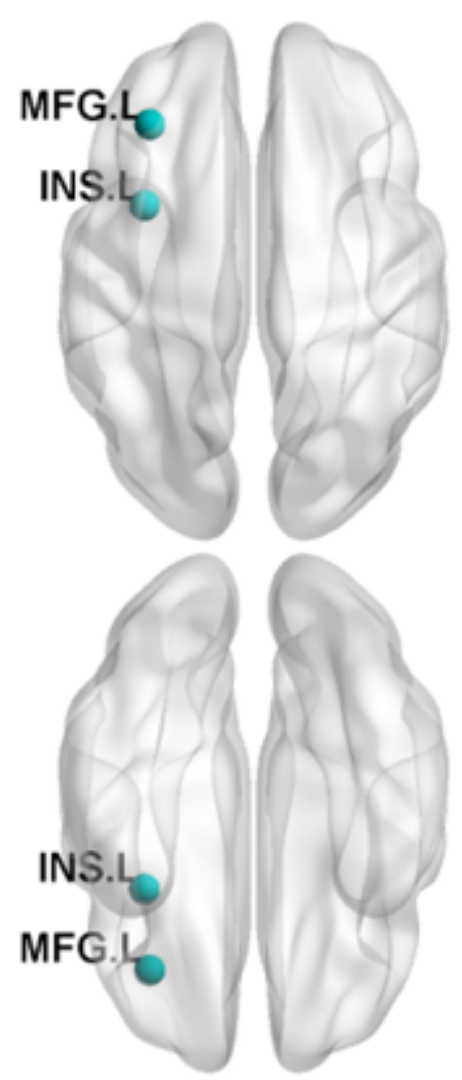
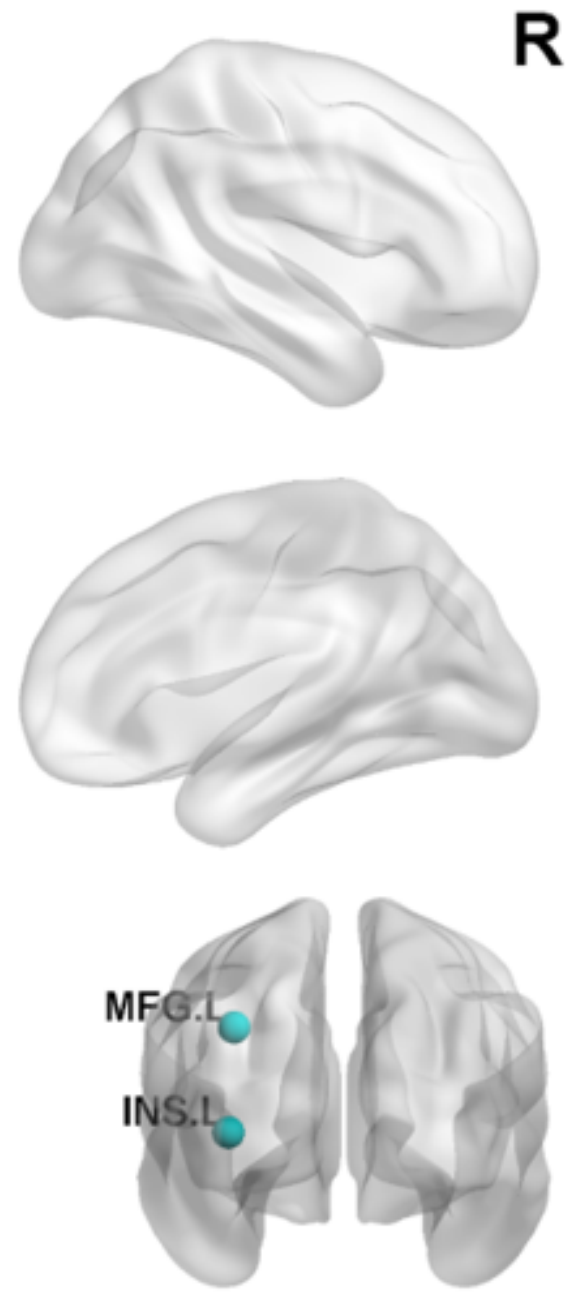

Figure 4

Regions with impaired nodal efficiency in CSVD+D group relative to CSVD-D group.

The regions impaired in efficiency are shown with blue nodes, with node size representing the betweengroup differences in nodal efficiency (Bonferroni corrected, $\mathrm{P}<0.05$ ).

Abbreviations: MFG.L represents left middle frontal gyrus, INS.L represents left Insula. 


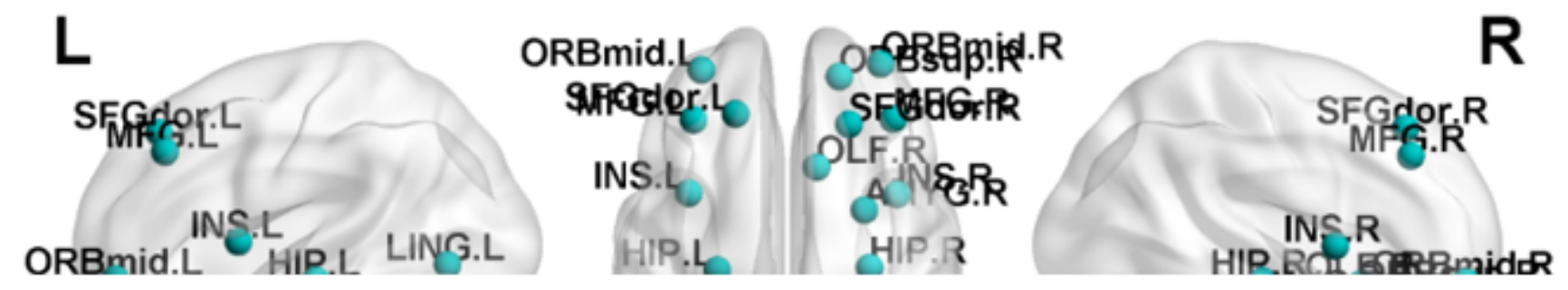

\section{Figure 5}

Regions with impaired nodal efficiency in CSVD+D group relative to NC group.

The regions impaired in efficiency are shown with blue nodes, with node size representing the betweengroup differences in nodal efficiency (Bonferroni corrected, $\mathrm{P}<0.05$ ).

Abbreviations: MFG.L and MFG.R represents bilateral middle frontal gyrus, ORBsup.L and ORBsup.R represents bilateral orbital middle frontal gyrus, INS.L and INS.R represents bilateral Insula, HIP.L and HIP.R represents bilateral hippocampus, ORBmid.L and ORBmid.R represents bilateral orbital middle frontal gyrus, SFGdor.L and SFGdor.R represents bilateral dorsolateral superior frontal gyrus, OLF.R represents right olfactory cortex, FFG.R represents right fusiform gyrus, LING.L represents left lingual gyrus, AMYG.R represents right amygdala. 


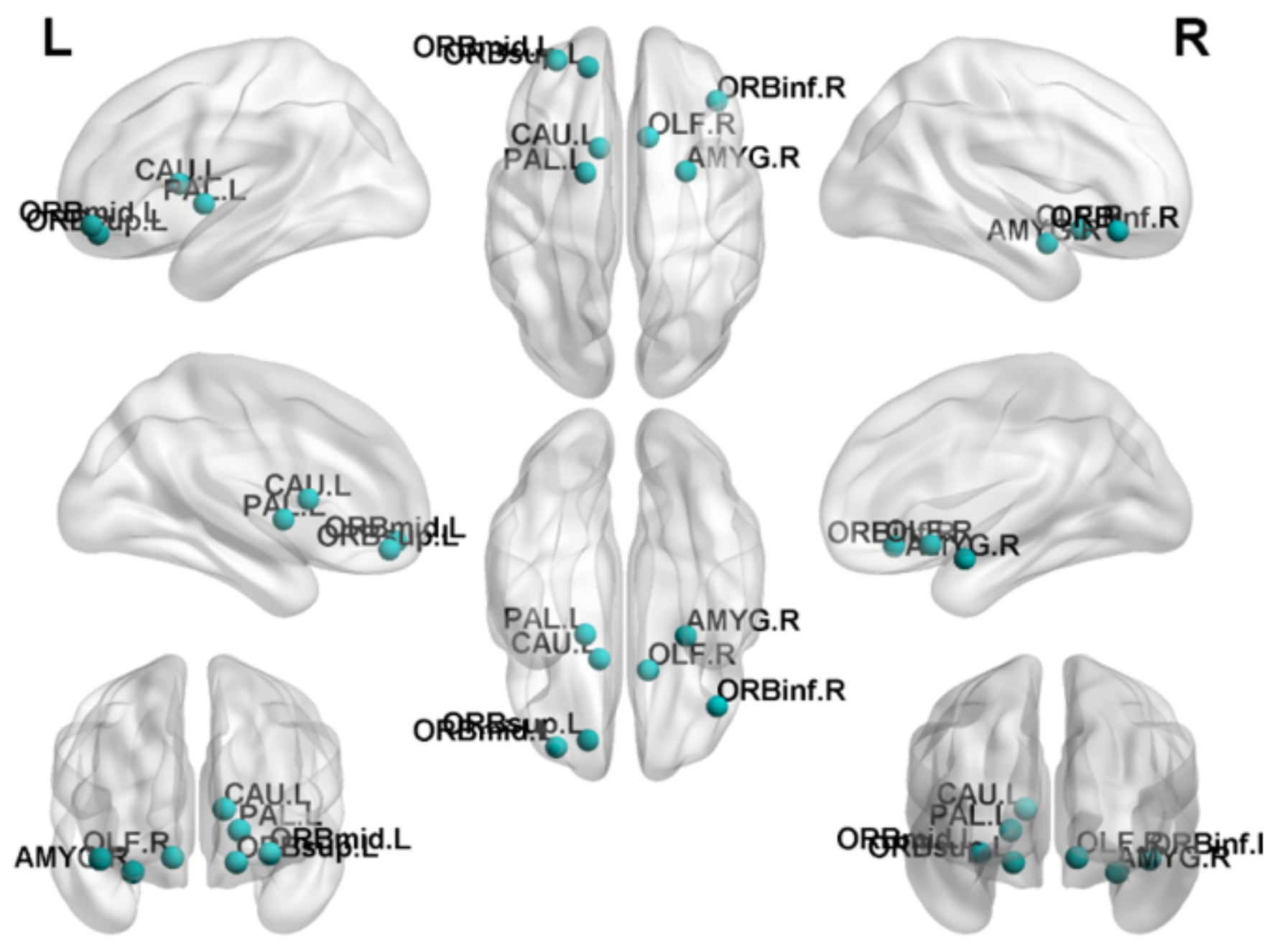

Figure 6

Regions with impaired nodal efficiency in CSVD-D group relative to NC group.

The regions impaired in efficiency are shown with blue nodes, with node size representing the betweengroup differences in nodal efficiency (Bonferroni corrected, $\mathrm{P}<0.05$ ).

Abbreviations: CAU.L represents left caudate nucleus, PAL.L represents left lenticular globus pallidus, ORBmid.L represents left orbital middle frontal gyrus, AMYG.R represents right amygdala, ORBsup.L represents left superior frontal gyrus of orbit, ORBinf.R represents right Inferior frontal gyrus of orbit, OLF.R represents right olfactory cortex. 